Check for updates

The BMJ

Cite this as: BMJ 2020;370:m3657 http://dx.doi.org/10.1136/bmj.m3657 Published: 17 September 2020

\section{Let pharmacists make minor substitutions in event of drug shortages, say stakeholders}

Clare Dyer

Groups representing doctors, pharmacists, and patients have written to England's health and social care secretary, Matt Hancock, calling for community pharmacists to be allowed to make minor substitutions for a GP's prescription in the event of drug shortages, particularly at the end of the transition period for Brexit.

The letter asking for a change in the law is signed by leaders of the BMA, the Royal College of General Practitioners, the Royal Pharmaceutical Society, the Pharmaceutical Services Negotiating Committee, Community Pharmacy Scotland, Community Pharmacy Wales, and the patients' group National Voices.

"The government has warned of potential disruption to medicines supplies at the end of the UK's transition period for exiting the European Union," the groups wrote. "Covid-19 has shown the importance of supporting and enabling professional judgment to put patients first, and this proposal should be part of prudent changes to enhance patient care. We would welcome your support and stand ready to work with your officials on how this could be implemented."

They noted that GPs and pharmacists were already facing drug shortages, but pharmacists are legally obliged to refer back to the prescriber before making even a minor adjustment. "This is frustrating for the patient, pharmacist, and prescriber," they wrote. "The process can cause delays in access to medicines and takes up health professionals' time, which could be used elsewhere to support patient care.”

They added, "Legislation should be amended to allow pharmacists to make minor amendments to a prescription, without a protocol, when a medicine is out of stock. Amendments would include changes such as a different quantity, strength, formulation or generic version of the same medicine (generic substitution)." The groups pointed out that such substitutions are routine for hospital pharmacists, that substitutions can be done in Scotland for drugs on a recognised shortages list, and that in Wales pharmacists may make certain changes without consulting the GP.

"These proposals would not extend to therapeutic substitution, which should instead be referred back to the prescriber, potentially notifying them of available alternatives to help them make appropriate changes to medicines," they said. "We recognise there are particular considerations for certain medicines, such as for epilepsy or transplants, and this change in policy should be supported with guidance on when substitutions may or may not be suitable. All of this should be underpinned by person centred care, clear communication, and decisions taken in discussion with patients."

A spokesperson for the health department said, "We have well established processes to deal with medicine shortages and work closely with the MHRA [Medicines and Healthcare Products Regulatory Agency], NHS England, and others operating in the supply chain to help prevent shortages and minimise any risks when they do arise. We will carefully consider the joint letter and the recommendations made."

A regulation that came into force last year allows the health and social care secretary to issue a serious shortage protocol when a shortage of a particular drug arises. Protocols, which are intended to be issued only in exceptional circumstances, specify the limited substitution the pharmacist may make. The groups say the protocols are rarely used. 